\title{
Determination of the frequency-dependent bulk modulus of glycerol using a piezoelectric spherical shell
}

\author{
T. Christensen and N. B. Olsen \\ Institue of Mathematics and Physics (IMFUFA), Roskilde University Center, Postbox 260, DK-4000 Roskilde, Denmark
}

(Received 23 February 1994)

\begin{abstract}
Through the coupling between the electrical capacitance of a spherical piezoceramic shell and the mechanical stiffness of a liquid contained therein, the frequency-dependent adiabatic bulk modulus $K_{s}(\omega)$ of the liquid can be derived. Using this method, $K_{s}(\omega)$ of glycerol in the range $15 \mathrm{~Hz}-15$ $\mathrm{kHz}$ has been measured at the glass transition. The loss peak frequencies of the compressibility $\kappa_{s}(\omega)=K_{s}^{-1}(\omega)$ and the specific heat $c_{p}(\omega)$ have the same temperature dependence, but the frequency dependences are different.
\end{abstract}

The relaxation processes in supercooled liquids at the glass transition are seen as a frequency dependence in many physical properties. The thermal relaxation and the scalar part of the mechanical relaxation are coupled and can be fully characterized by three independent relaxation functions. Let $s$ be the entropy density, $T$ the temperature, $\epsilon_{i j}$ the strain tensor, and $\sigma_{i j}$ the stress tensor. We denote the relative volume change by $\epsilon=\operatorname{Tr}\left(\epsilon_{i j}\right)$ and the hydrostatic pressure by $p=-\frac{1}{3} \operatorname{Tr}\left(\sigma_{i j}\right)$. Let $d s$, $d \epsilon, d T, d p$ be the amplitudes of harmonically varying small perturbations $\propto e^{-i \omega t}$. Then one has

$$
\left(\begin{array}{c}
d s \\
d \epsilon
\end{array}\right)=\left(\begin{array}{cc}
\frac{1}{T} c_{p} & \alpha_{p} \\
\alpha_{p} & \kappa_{T}
\end{array}\right)\left(\begin{array}{c}
d T \\
-d p
\end{array}\right)=J\left(\begin{array}{c}
d T \\
-d p
\end{array}\right)
$$

where $c_{p}(\omega)$ is the isobaric specific heat, $\alpha_{p}(\omega)$ is the isobaric expansion coefficient, and $\kappa_{T}(\omega)$ is the isothermal compressibility. These three quantities constitute the thermoelastic compliance matrix $J$. The quantities are complex and thereby also describe the phase shift introduced by the relaxation processes. ${ }^{1}$ How these relaxation functions are related is still an open question. Zwanzig ${ }^{2}$ has proposed a model in which the normalized relaxation functions of $c_{p}(\omega)$ and $\kappa_{T}(\omega)$ are identical. However, here we consider the adiabatic compressibility $\kappa_{s}(\omega)$, which is more convenient to measure. The triple $\kappa_{s}(\omega), c_{p}(\omega)$, and $\alpha_{p}(\omega)$ will also contain the full information on $J$.

What is known experimentally about these functions? Consider glycerol, the canonical example of the glass transition. Nothing seems to be known about $\alpha_{p}(\omega)$. Different techniques have given very different results on $c_{p}(\omega)$. The one method, ${ }^{3}$ which could cover a wide frequency range $\left(10^{-2}-3 \times 10^{4} \mathrm{~Hz}\right)$, found that the normalized specific heat $c_{n}(\omega)=\left[c_{p}(\omega)-c_{p}(\infty)\right] /\left[c_{p}(0)-c_{p}(\infty)\right]$ could be fitted to $1-\Phi_{\beta}(\omega \tau)$, where

$$
\Phi_{\beta}(\tilde{\omega})=-i \tilde{\omega} \int_{0}^{\infty} e^{-u^{\beta}} e^{i \tilde{\omega} u} d u
$$

is the frequency domain function corresponding to the Kohlrausch-William-Watts relaxation function. These authors found $\beta=0.65 \pm 0.03$ (KWW exponent). The high-frequency behavior was thus $\omega^{-0.65}$, very far from the behavior $\omega^{-0.28}$ found by the other method. ${ }^{4}$ The latter result relied on the use of the temperature-time superposition principle (TTSP), which the first method seemed to show valid.

The bulk modulus $K_{s}(\omega)$ was deduced from ultrasonic measurements ${ }^{5}$ of the longitudinal $c_{1}$ and the transversal $c_{t}$ sound velocity and $K_{s}(\omega)$ was described by a ColeDavidson distribution of relaxation times. That is, the normalized bulk modulus was given by $1-(1-i \omega \tau)^{-\beta}$ with $\beta_{\mathrm{CD}}=0.32$. The result was based on a few frequencies in the range 1-100 $\mathrm{MHz}$ using the TTSP. The asymptotic behavior of the compressibility $\kappa_{s}(\omega)\left[=K_{s}^{-1}(\omega)\right]$ in the high-frequency limit was thus $\omega^{-0.32}$, close to the behavior of $c_{p}(\omega)$ found by Christensen. ${ }^{4}$ More recent ultrasonic measurements ${ }^{6}$ - still relying on TTSP-have given $\beta_{\mathrm{KWW}}=0.60 \pm 0.05$ for the longitudinal compliance $M_{s}^{-1}(\omega)$. Jeong, Nagel, and Bhattacharya found that this KWW exponent and the one in the $c_{p}(\omega)$ experiment of Birge and Nagel were within experimental error of each other. However, $M_{s}(\omega)=K_{s}(\omega)+\frac{4}{3} G(\omega)$, and it is not given a priori that $K_{s}(\omega)$ and the shear modulus $G(\omega)$ relax in the same way. In fact, Piccirelli and Litovitz found that $G$ was described by a logarithmic symmetrical Gaussian distribution of relaxation times in contrast to the asymmetrical Cole-Davidson distribution describing $K_{s}$. It is thus of interest to measure the pure bulk modulus $K_{s}(\omega)$ and to measure it in the frequency range of the $c_{p}(\omega)$ experiments below the ultrasonic regime.

We have developed a method capable of this and applied it to glycerol. The method operates at low frequencies, i.e., frequencies at which the corresponding acoustic wavelength is much larger than the sample size (quasistatic regime). On the other hand the frequencies are sufficiently high to ensure adiabatic conditions, i.e., the corresponding thermal diffusion length is much smaller than the sample size. The relaxation function can be found without using the TTSP, since the frequency can be scanned continously. Furthermore, our method also determines $M_{s}$ at certain discrete resonance frequencies in the ultrasonic regime. The method is based on the coupling between the electrical capacitance of a spherical piezoceramic shell and the mechanical stiffness of a liquid contained therein. There are no separate emitter and receiver of acoustic vibrations, only one transducer, which constitute the sample cell also. The shell is made 
of a piezoceramic material (pz26, Ferroperm, Denmark) polarized in the radial direction. We will call it the piezoelectric bulk modulus gauge (PBG). It is covered with electrodes on the inner and outer surfaces. On applying a potential difference across the shell, the PBG will expand or contract radially depending on the polarity. For a mechanical free outer surface, the coupling between the complex amplitudes of the normal stress $\sigma$ and the volume change $\Delta V$ on the one hand, and the surface charge $Q$ and the potential difference $U$ on the other hand, is given by a transfer matrix $c_{i j}$,

$$
\left(\begin{array}{c}
U \\
Q
\end{array}\right)=\left(\begin{array}{ll}
c_{11} & c_{12} \\
c_{21} & c_{22}
\end{array}\right)\left(\begin{array}{c}
\sigma \\
\Delta V
\end{array}\right)
$$

The measured electrical capacitance therefore depends on whether the shell is free to move $(\sigma=0)$ or clamped $(\Delta V=0)$,

$$
C_{\text {free }}(\omega)=\frac{c_{22}}{c_{12}}, \quad C_{\text {clamped }}(\omega)=\frac{c_{21}}{c_{11}} .
$$

If a medium of stiffness $S(\omega)=\sigma / \Delta V$ is placed inside the PBG, then the electrical capacitance becomes

$$
C(\omega)=\frac{c_{22}+c_{21} S}{c_{12}+c_{11} S}
$$

Thus, $S(\omega)$ can be found knowing $c_{i j}$ and measuring $C(\omega)$. For a thin piezoelectric ceramic shell, $c_{i j}$ can be expressed by the inner radius $r$, thickness $t$, density $\rho$, elastic constants $s_{11}, s_{12}$, piezoelectric constant $d_{13}$, and dielectric constant $\epsilon_{33} .{ }^{7}$ Introducing the "breathing mode" resonance frequency

$$
\omega_{c}=\frac{1}{r}\left(\frac{2}{\left(s_{11}+s_{12}\right) \rho}\right)^{1 / 2}
$$

the planar coupling constant $k_{p}=\left[2 d_{13}^{2} /\left(s_{11}+\right.\right.$ $\left.\left.s_{12}\right) \epsilon_{33}\right]^{1 / 2}$, the free capacitance at zero frequency $C_{0}=$ $C_{\text {free }}(0)=\left(4 \pi r^{2} / t\right) \epsilon_{33}$, and the inertance $L=\rho t / 4 \pi r^{2}$, the result is

$$
\left(c_{i j}\right)=\left(\begin{array}{cc}
\frac{1}{k_{p} \omega_{c} \sqrt{L C_{0}}} & \frac{\omega_{c}}{k_{p}}\left(\frac{L}{C_{0}}\right)^{1 / 2}\left[1-\left(\frac{\omega}{\omega_{c}}\right)^{2}\right] \\
\frac{1-k_{p}^{2}}{k_{p} \omega_{c}}\left(\frac{C_{0}}{L}\right)^{1 / 2} & \frac{\omega_{c}}{k_{p}} \sqrt{L C_{0}}\left[1-\left(1-k_{p}^{2}\right)\left(\frac{\omega}{\omega_{c}}\right)^{2}\right]
\end{array}\right) .
$$

In the specific case $\rho=7.65 \mathrm{~g} \mathrm{~cm}^{-3}, t=0.10 \mathrm{~cm}, r=$ $0.90 \mathrm{~cm}$, whereby $L=7.52 \times 10^{-2} \mathrm{~g} \mathrm{~cm}^{-4}$. By fitting a measurement of the free electrical capacitance to the theoretical expression

$$
C_{\text {free }}(\omega)=\frac{c_{22}}{c_{12}}=C_{0} \frac{1-\left(1-k_{p}^{2}\right)\left(\omega / \omega_{c}\right)^{2}}{1-\left(\omega / \omega_{c}\right)^{2}}
$$

the three constants $C_{0}, k_{p}, \omega_{c}$ are found. These constants are both temperature and weakly time dependent due to annealing processes in the piezoceramic itself. Thus the same time and temperature scheme is exactly followed during reference measurement and modulus mea- surement. Typical values are $C_{0}=12 \mathrm{nF}, k_{p}=0.51$, $f_{c}=\omega_{c} / 2 \pi=85 \mathrm{kHz}$. One has to correct the expression (5) for the transfer matrix and take the finite thickness of the transducer into account. We have indeed calculated and used the general transfer matrix, but these lengthy expressions are omitted here. The corrections amounts to $15 \%$ on $K_{s}$.

At the top of the sphere a small hole of radius $r_{h}$ makes it possible to fill the sphere with liquid. Also, a reservoir of liquid resides in a small tube on top of the sphere. The hole connects this to the inside of the sphere, allowing for thermal expansion of the liquid. Despite this hole, the liquid is virtually confined in the sphere at the frequencies of interest. The characteristic time scale of the glass transition is given by the Maxwell relaxation time $\tau_{M}=\eta_{0} / G_{\infty}$, where $\eta_{0}$ is the low-frequency limit of the viscosity and $G_{\infty}$ is the high-frequency limit of the shear modulus. Assuming Poiseuille flow through the hole, a characteristic flow time $\tau_{f}$ will be

$$
\tau_{f}=\frac{32}{3} \frac{r^{3} t}{r_{h}^{4}} \frac{\eta}{K_{s}} \approx 10^{5} \tau_{M}
$$

Thus, one has in fact quite a large range of times beyond the Maxwell relaxation time at disposal. On the other hand, the cell can of course only be filled in a reasonable time at high temperatures where the viscosity is low.

The stiffness $S(\omega)$ of a spherical isotropic viscoelastic solid is derivable from the solution of the equation of motion. ${ }^{8}$ If the density is $\rho_{l}$, the longitudinal wave vector $k_{l}=\sqrt{\left(\rho_{l} / M_{s}\right)} \omega$, and the volume $V=\frac{4}{3} \pi r^{3}$, then one finds

$$
S(\omega)=\frac{1}{V}\left\{K_{s}-M_{s}\left(1+\frac{1}{3} \frac{\left(k_{l} r\right)^{2} \sin \left(k_{l} r\right)}{k_{l} r \cos \left(k_{l} r\right)-\sin \left(k_{l} r\right)}\right)\right\}
$$

At low frequencies $S(\omega)$ is simply $K_{s}(\omega) / V$. At higher frequencies it depends on both $K_{s}(\omega)$ and $M_{s}(\omega)$ because longitudinal waves are excited.

The method was applied to that canonical example of the glass transition, glycerol. Figure 1 shows how the electrical capacitance $C(\omega)$ of the PBG is reduced from its free value by the partial clamping of the transducer due to the contained liquid. The glass transition in this picture is seen indirectly in the decrease of $C$ with increasing frequency. Also shown is the measured $C_{\text {free }}$ and the calculated $C_{\text {clamped }}$.

Figures 2 and 3 present the measured real and imaginary part of bulk modulus $K_{s}$ as a function of frequency at different temperatures. Denote the normalized bulk modulus by $K_{n}(\omega)=\left[K_{s}(\omega)-K_{0}\right] /\left(K_{\infty}-K_{0}\right)$. The solid line represents a fit to data of a phenomenological model, where $K_{n}(\omega)$ is given by an extended Maxwell model $K_{n}(\omega)=\left[1+\left(-i \omega \tau_{b}\right)^{-1}+q\left(-i \omega \tau_{b}\right)^{-\alpha}\right]^{-1}$. It is found that $q=1.40 \pm 0.03$ and $\alpha=0.43 \pm 0.02$. $\tau_{b}$ is temperature dependent and corresponds to the Maxwell relaxation time. The best fit of data to a frequency domain Kohlrausch function $\Phi_{\beta}$ gives $\beta=0.43 \pm 0.02$. Such a fit is almost as good as the extended Maxwell fit.

It seems natural in a comparison of the thermal and 


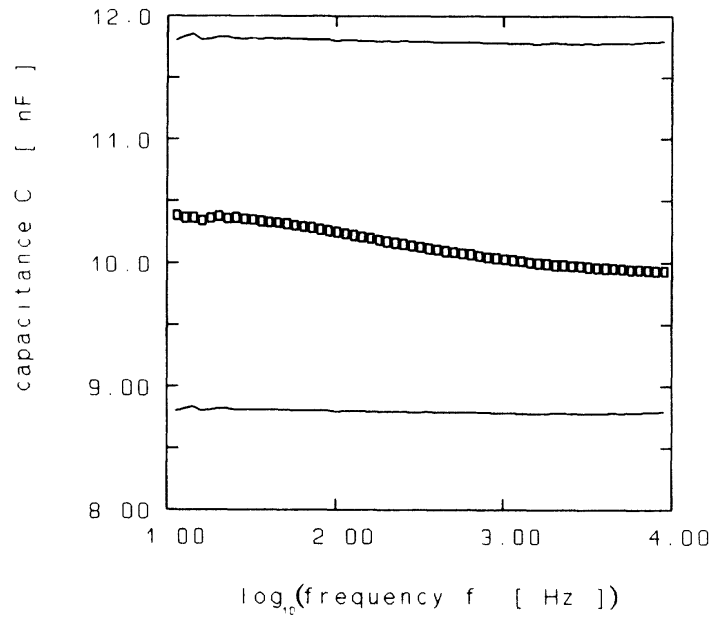

FIG. 1. The electrical capacitance of the PBG filled with glycerol at $210 \mathrm{~K}(\square)$. Upper solid line is $C_{\text {free }}$. Lower solid line is $C_{\text {clamped }}$.

mechanical relaxation to compare the generalized compliance functions, ${ }^{6}$ although this is not forced by any general principle. The compliance functions in this case are $c_{p}(\omega)$ and $\kappa_{s}(\omega)$. The loss peak frequency $f_{p}$ of the compressibility can be fitted to $f_{p}=f_{0} \exp \left[-\left(T / T_{0}\right)^{3}\right]$ with $f_{0}=6.67 \times 10^{12} \mathrm{~Hz}$ and $T_{0}=612 \mathrm{~K}$ using the same functional form as Piccirelli and Litovitz. ${ }^{5} \tau_{b}$ is $0.126 f_{p}^{-1}$ in the fitting model for the present value of $q$ and $\alpha$. The loss peak frequency of $\kappa_{s}$ and $c_{p}$ (Ref. 3) can be compared in a temperature interval 202-226 K, where they both are measured. While the loss peak frequencies vary 4 orders of magnitude, their ratio is rather constant, $f_{p}\left(\kappa_{s}\right) / f_{p}\left(c_{p}\right) \approx 5$. This common temperature dependence shows a connection between the thermal and mechanical relaxation. However, we find that the shapes of the relaxation functions are not alike. The normalized compressibility $\kappa_{n}=\left(K_{s}^{-1}-K_{\infty}^{-1}\right) /\left(K_{0}^{-1}-K_{\infty}^{-1}\right)$ cannot be described by a Kohlrausch fit of the type $1-\Phi_{\beta}$ for any $\beta$ in contrast to the $c_{p}(\omega)$ data of Birge and Nagel. This also follows from the relation between $\kappa_{n}$ and $K_{n}$,

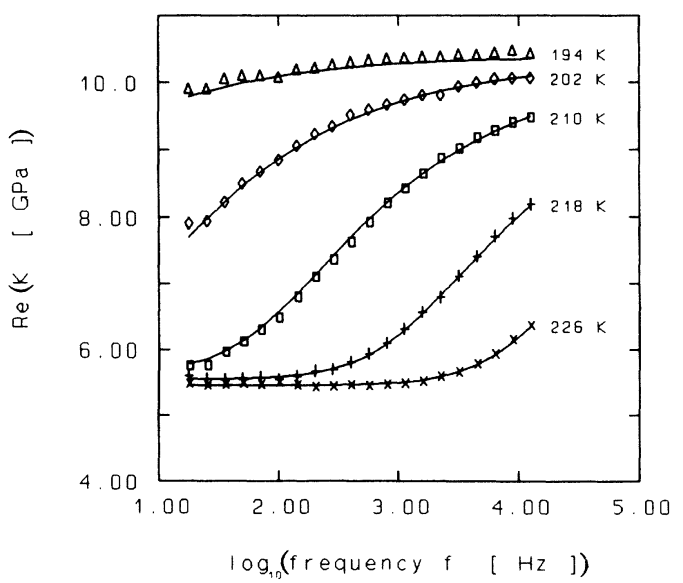

FIG. 2. The real part of bulk modulus of glycerol at the glass transition as a function of frequency at different temperatures. The solid line is a fit to an extended Maxwell model.

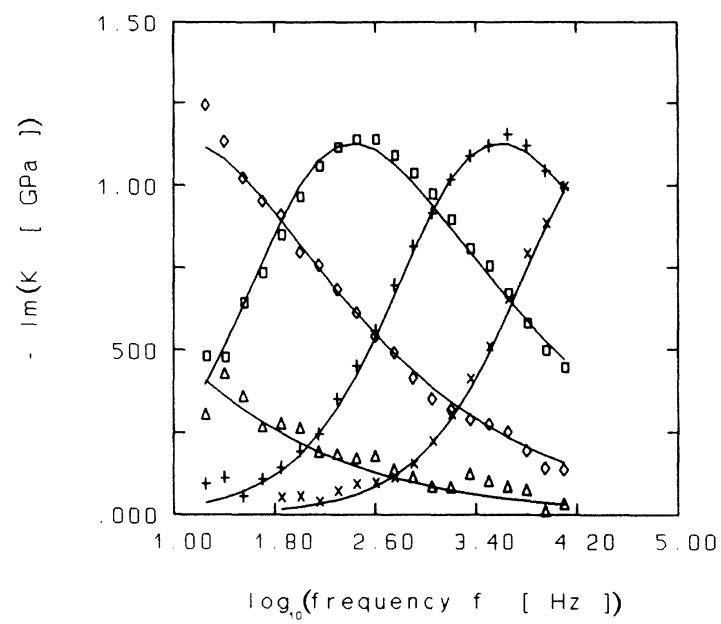

FIG. 3. The imaginary part of bulk modulus corresponding to the real part shown in Fig. 2.

$1-\kappa_{n}=(1+A) K_{n} /\left(A+K_{n}\right)$, where $A=K_{0} /\left(K_{\infty}-K_{0}\right)$ $(=1.2)$. Since $K_{n}$ nearly had the Kohlrausch form, this cannot be the case for $1-\kappa_{n}$. In general the Kohlrausch function may be used in a modulus, or a compliance formalism, but not for the same physical property (unless $\beta=1) . \kappa_{n}(\omega)$ does not match the $c_{p}(\omega)$ of Christensen either. These data were, however, found at lower temperatures and may be uncertain due to the use of the TTSP. In Fig. 4 the fitting functions of the three experiments are shown in an Argand diagram. The differences are clearly outside the scatter of the original data around the fitting functions.

The real and imaginary part of the specific stiffness $S(\omega) V$ of the liquid sphere as a function of temperature at $1 \mathrm{kHz}$ is shown in Fig. 5. Two dispersion regions are seen with maxima in the imaginary part at 214 and $268 \mathrm{~K}$, respectively. The low-temperature dispersion region is simply the glass transition in $K_{s}$, since at this frequency the measured specific stiffness $(8)$ is equal to the bulk modulus. At higher temperature the liquid is able to flow through the filling hole and this give rise to the second dispersion region. Therefore, although the measured stiffness still reflects relaxation processes at the glass transition, it does not give bulk modulus and can-

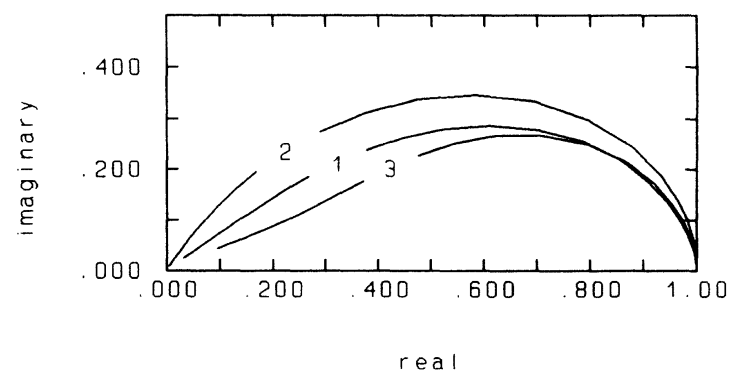

FIG. 4. Argand diagram of the functions used in the fit of data to (1) the adiabatic compressibility $\kappa_{s}(\omega)$ (this work), (2) the isobaric specific $c_{p}(\omega)$ of Ref. 3, and (3) $c_{p}(\omega)$ of Ref. 


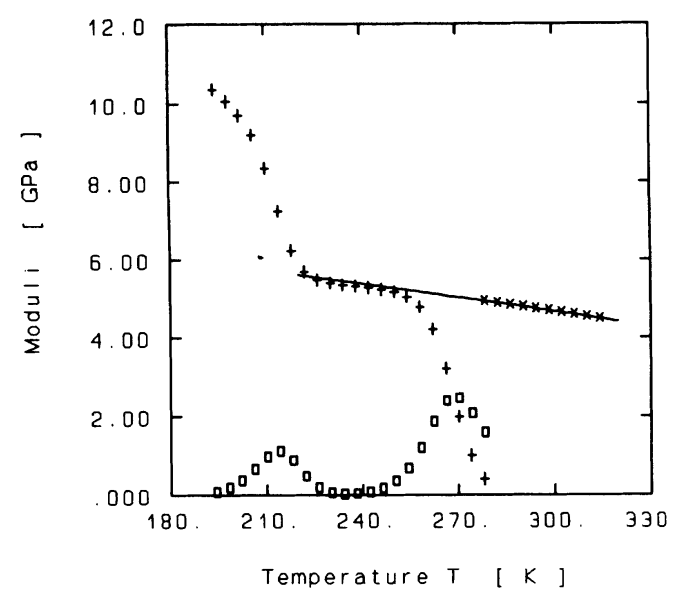

FIG. 5. Real (+) and imaginary ( $\square$ ) parts of the specific stiffness at $1 \mathrm{kHz}$ measured by the quasistatic method. $K_{0}$ $(\times)$ measured by the resonance method.

not easily be analyzed in a rigorous way. The ratio of $f_{p}$ at 214 and $268 \mathrm{~K}$ is $10^{5}$, which is in agreement with the estimate (7).

According to (8) a viscoelastic sphere will show stiffness resonances when $\tan \left(k_{l} r\right)=k_{l} r$. These resonances are seen in the electrical capacitance of the PBG. Although the resonances are moved due to the mechanical coupling of the PBG and the liquid, this is only of importance for the lowest lying resonances. Thus for resonance frequency $\nu_{n}, n \geq 3$, the condition simply gives longitudinal modulus to a good approximation as $16(1+2 n)^{-2} \rho_{l} \nu_{n}^{2} r^{2}$.

In Fig. $5 M_{s}$ calculated by the third resonance at $280-320 \mathrm{~K}$ is shown. At these temperatures the inverse Maxwell relaxation time is much higher than the resonance frequency. Thus shear modulus can be neglected compared to bulk modulus and so $M_{0}=K_{0}$ in this limit. The extrapolation of $K_{0}(T)$ measured by this resonance technique into the temperature region, where the quasistatic method works, agrees within $1 \%$. In this way one has an independent and simple check on the validity of the procedure of the quasistatic method.

In conclusion, we have developed a method for measuring the pure frequency-dependent adiabatic bulk modulus. This method was applied to glycerol in a temperature and frequency range which enables direct comparison with the specific-heat measurements of Birge and Nagel. ${ }^{3}$ Although the loss peak frequency of $\kappa_{s}$ and $c_{p}$ have the same temperature dependence and therefore the thermal and mechanical relaxation must be connected somehow, the relaxation functions have different shapes. However a common relaxation mechanism need not lead to the same normalized relaxation function of $\kappa_{s}$ and $c_{p}$. It could be that a comparison of $\kappa_{T}$ and $c_{p}$ should be made instead as suggested by Zwanzig. ${ }^{2}$ However, in order to convert data, information on a third thermoelastic response function, e.g., $\alpha_{p}(\omega)$, would be necessary.
${ }^{1}$ J. Meixner and H. G. Reik, in Prinzipien der Thermodynamik und Statistik, edited by S. Flügge, Handbuch der Physik, Gruppe 2, Band 3, Teil 2 (Springer, Berlin, 1959) pp. 482-485.

2 R. Zwanzig, J. Chem. Phys. 88, 5831 (1988).

${ }^{3}$ N. O. Birge and S. R. Nagel, Phys. Rev. Lett. 54, 2674 (1985).

4 T. Christensen, J. Phys. (Paris) Colloq. 46, C8-635 (1985).

${ }^{5}$ R. Piccirelli and T. A. Litovitz, J. Acoust. Soc. Am. 29, 1009 (1957).
${ }^{6}$ Y. H. Jeong, S. R. Nagel, and S. Bhattacharya, Phys. Rev. A 34, 602 (1986).

${ }^{7}$ D. A. Berlincourt, D. R. Curran, and H. Jaffe, in Methods and Devices, edited by W. P. Mason, Physical Acoustics: Principles and Methods Vol. 1A (Academic, New York, 1964), p. 224.

${ }^{8}$ L. D. Landau and E. M. Lifshitz, Elasticity Theory, 3rd ed., Course of Theoretical Physics Vol. 7 (Pergamon, New York, 1986), pp. 87-91. 